\title{
Construction for obtaining trellis run length limited error control codes from convolutional codes
}

\author{
Peter Farkaš ${ }^{*}$, Frank Schindler ${ }^{* *}$
}

\begin{abstract}
Recently a new construction of run length limited block error control codes based on control matrices of linear block codes was proposed. In this paper a similar construction for obtaining trellis run length limited error control codes from convolutional codes is described. The main advantage of it, beyond its simplicity is that it does not require any additional redundancy except the one which is already contained in the original convolutional error control code. One example is presented how to get such a code from a convolutional low density parity check code.
\end{abstract}

K e y w o r d s: run length limited code error correcting code, convolutional code, convolutional low density parity check code, trellis run length limited error control code

\section{Introduction}

Run length limited (RLL) codes are used in communications and storage systems in order to fulfill numerous practical requirements $e g$ to support synchronization or decrease DC component in signals or adapt the spectral characteristics of the signal to a channel or others. In digital domain the functions and constructions of a RLL codes are closely related to channel model, which describes these requirements in exact form. The most common channel models are state diagram, trellis and incidence matrix [1]. In theoretical considerations it is supposed that the model of the constrained channel is errorless [1]. It is possible to specify the tasks of RLL encoder and decoder very simply in connection with such errorfree constrained channel model.

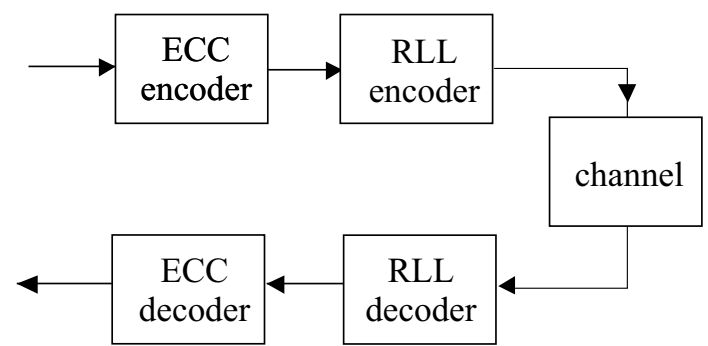

Fig. 1. Concatenation of an inner RLL encoder/decoder and the outer ECC encoder/decoder used in most practical applications

Namely, the task of the RLL encoder is to translate an arbitrary sequence of digital symbols, which comes to its input to a sequence on its output which fulfills the channel constraints. The task of the decoder is to translate the sequence received from a channel into a sequence which is identical to the original sequence on the input of the encoder. It is obvious that in practice this task is complicated by the fact that a real noisy channel could cause errors in the received sequence. Therefore, in practical applications, error control code (ECC) is used in cascade with RLL code usually as illustrated in Fig. 1.

However this concatenation is problematic because the inner RLL decoder cannot correct errors and in some cases it can even cause error propagation [2]. This can seriously decrease the performance of the overall system. Therefore it is advantageous to use a combined run length limited error control code(RLL-ECC). This could be obtained by incorporating the error correction capabilities into the RLL code or by incorporating RLL properties into an ECC or constructing the RLL-ECC from a scratch by other methods.

Let us shortly describe the state of the art in the construction techniques area of trellis RLL-ECCs ( $t-R L L-$ ECCs). (For block RLL-ECC codes it could be found in [3].) Trellis RLL codes and trellis ECCs were and are used for long in practical applications. The first RLL trellis codes were simple line codes such as Non Return to Zero Inverted (NRZI) and Miller Code. NRZI line code was applied for example in 9 track magnetic tape data storage for IBM System 360. More recent example is from [4], where it was proposed to use the Miller code in concatenation with another convolutional code for visible light communication. The idea to combine RLL and ECC codes was for the first time proposed in [5]. So called combined correction/modulation codes (CCMC) were introduced there. The first construction presented in [5] is based on exploiting set partitioning and the second on using convolutional codes together with precoders. Other

* Faculty of Electrical Engineering and Information Technology, Slovak University of Technology, Ilkovičova 381219 Bratislava, Slovakia, email: p.farkas@ieee.org, ** Faculty of Informatics, PanEuropean University, Tematínska 10,85105 Bratislava, Slovakia 


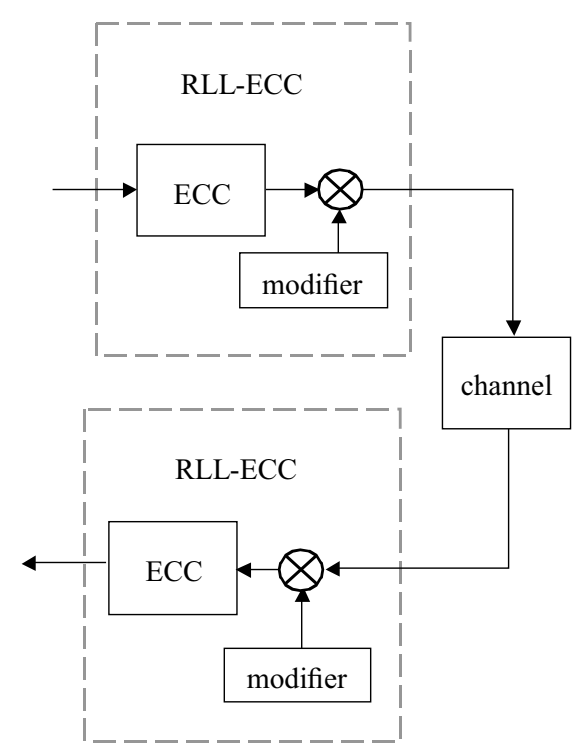

Fig. 2. Illustration how a modifier could be used in order to obtain an RLL-ECC from ECC without adding any redundancy and without requiring the ECC decoder to be modified.

constructions for obtaining CCMCs could be found in [6]. In [7] it was proposed to modify linear binary block codes into RLL-ECCs without introducing of additional redundant symbols. The construction in [7] exploits two particular properties, which are present in generator matrix G of some linear binary codes and a binary vector $\mathbf{m}$ called the modifier, which is added to each codeword. This technique was applied later on convolutional codes in [9] and on TurboCodes in [10] and [11] in order to obtain trellis RLL-ECCs. In recent time low density parity check (LDPC) codes started to be very popular for practical applications thanks to their excellent error correction capabilities. However in practical systems, which behind error correcting capabilities require also RLL properties, they were used mostly in concatenation arrangement depicted in Fig. 1, [11-14]. The first attempt to incorporate the RLL properties into block LDPC codes was done in [15]. In [15] bit flipping (error insertion) was used to fulfill RLL constraints, but at the cost of deteriorating the error correction capabilities of the underlying LDPC codes because each flipped bit was treated as error. This means it had to be corrected and as a consequence the number of correctable errors which occurred in channel was decreased. The method was later improved gradually in [16-18] up to the stage that the error control deterioration caused by bit flipping was eliminated on the expense of quite complicated decoding techniques. Recently new construction method for obtaining RLL-ECCs from linear binary block ECCs was proposed in [19]. In this short communication it is shown that this method could be adapted also for obtaining trellis t-RLL-ECCs from convolutional ECCs (c-ECC). More specifically it could be applied to transform any c-ECC which has a special property in its control matrix into the t-RLL-ECC. However for practical applications probably the most attractive consequence is that also c-LDPC codes, which possess this property in their control matrices, could be transformed into t-RLL-ECCs. Therefore the construction is explained in this manuscript using one c-LDPC as an illustrative example.

\section{High level description of the method}

The basic idea which allows obtaining RLL-ECCs from binary LBC-ECCs is illustrated in Fig. 2.

After the message is encoded by the binary ECC, a specific fixed binary vector $\mathbf{m}$, called the modifier, is added to each codeword. The result of this addition is that the positions corresponding to ones in a modifier will be inverted in each codeword. This addition does not introduce any additional redundancy into the encoded codeword and the inversions could be eliminated by a second addition of the same modifier to the transmitted word at the input of the receiver. The consequence is that the original decoder used for the underlying ECC does not have to be modified. This simple idea was proposed in [7] and was based on two properties which could often be found in generator matrix of LBC ECC. Unfortunately some codes are not specified by generator matrices. For example the most advanced codes used nowadays in many standards namely the LDPC codes and c-LDPC codes are defined by their control matrices $\mathbf{H}$. It means that the methods based on generator matrices properties observed in [7] could not be applied directly to such codes. In [19] another property was proposed as an alternative, which could be observed in many $\mathbf{H}$ matrices in binary LBSECCs. Namely, if an even number of ones is discovered in a row of a control matrix in a set of positions $\Re$, then negating an odd number of corresponding positions in a codeword will cause these positions to not all be the same. In other words they will not contain all ones or all zeros. Because of this, the resulting codeword will have RLL properties. We will show that the same property could also be used for binary convolutional codes and so also for $\mathrm{C}-\mathrm{LDPC}$ codes.

\section{Trellis RLL-ECC obtained based on control matrices}

The simplest way of explaining the new construction of t-RLL-ECC is to use an example. Convolutional ECC could be specified via their control matrices. In contrast to control matrices of block codes, these control matrices are infinite. Typically they have nonzero entries only in a region around a diagonal, which has a form similar to a stair case. In Fig. 3 there is an example of a c-LDPC code obtained by unwrapping a code constructed by Gallager [20] using the method proposed in [21].

It is obvious that a modifier for such a code also has to be infinite in case we would like to use a method depicted in Fig. 2. One can observe that the code is a regular cLDPC code with 3 ones and 4 ones in each column and 


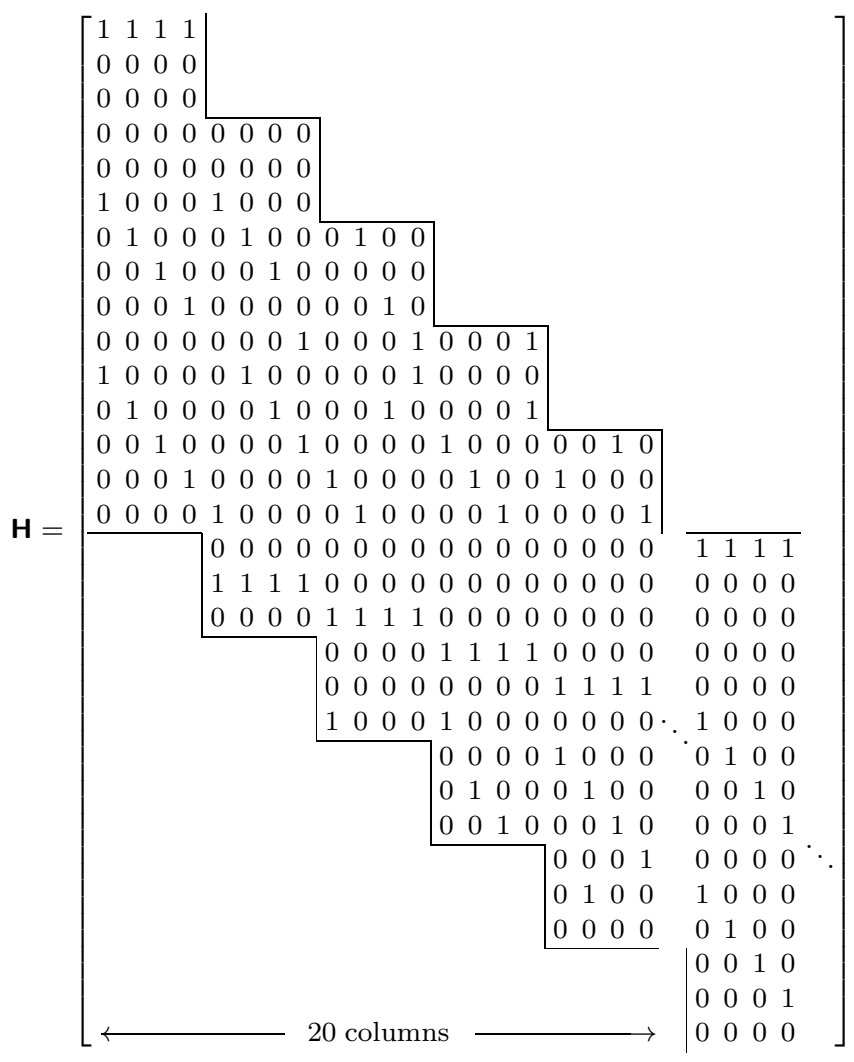

Fig. 3. Convolutional regular LDPC code control matrix obtained using construction [21] from a Gallager LDPC code published in [20]. The same staircase structure of the first 20 columns repeats itself periodically, empty spaces, outside staircase-like border contain zeros.

row respectively. From this it follows that each row in its H contains a set with 4 ones. In other words, each row specifies one control equation in which 4 symbols of the codeword take part. However each symbol is contained in 3 different control equations. Therefore it is necessary to choose nonoverlapping subsets of intervals, which cover some of the control equations or, more precisely, the corresponding subsets of symbols, which take part in them and which are between them in a row. We will call these intervals a row spans. Because the goal is to select an odd number of negations in each such span to get an infinite modifier, it is appropriate to find a rule, which will allow specify the positions of ones in the modifier without to write it down. For example, a periodic rule could be a convenient way to do it. On the other hand it would be appropriate to choose the intervals covering symbols participating in control equations as short as possible and, if possible, without any gaps between them in order to get minimal lengths of runs with identical symbols. In the matrix depicted in Fig 3 consecutive quadruples of ones are in rows (given in order corresponding to a sliding window with 20 columns): 1, 17, 18, 19, 20, 16, 32, 33, 34, 35, $31,47,48,49,50$. These observations and requirements should be considered in the approach to construct an appropriate modifier. Applying them in our example, the appropriate modifier could have an odd number of ones in each block of 4 bits. For example

$$
\mathbf{m}=(1,0,0,0,1,0,0,0,1,0,0,0,1,0,0,0,1,0,0,0,1, \ldots)
$$

Adding modifier (1) to the infinite codeword in practice means that the first and then each following fourth symbol coming from a encoder output should be inverted. This simple operation will result in the longest run of identical symbols in a codeword at most 6 bits long. In the receiver the same positions should be negated and therefore a synchronizing the inversions, or in other words, the modifier is necessary. In case that the inversions will be done in synchronism, then no modification of the c-LDCP decoder will be needed.

\section{Conclusions}

In this manuscript a method was proposed which could be used to construct trellis RLL-ECCs. It was illustrated using an example in which a t-RLL-ECC was obtained from a c-LDPC code without introducing any additional redundancy into the encoded sequence. The method could be used with any c-ECC which is defined by a control matrix, which possesses rows with even number of ones in them.

This work was supported by the European Space Agency (Contract No: 4000117400/16/NL/NDe) and the Grant Agency Academic Alliance (grant GA/2016/10). 


\section{REFERENCES}

[1] R. E. Blahut, Digital Transmission of Information, Addison Wesley, 1990.

[2] P. Lee and J. K. Wolf, "Combined Error Correction/Modulation Codes" IEEE Transactions on Magnetics, vol. 23 no. 5, Sep 1987, pp. 3681-3683,

[3] K. Farkašová, et al "Construction of Error Control Run Length Limited Codes Exploiting some Parity Matrix Properties", Journal of Electrical Engineering vol. 66, no. 3, March 2015, pp. $182-184$.

[4] X. Lu and J. Li Tiffany, "Achieving FEC and RLL for VLC: A Concatenated Convolutional Miller Coding Mechanism" IEEE Photonics Technology Letters, vol. 28 no. 9, May 2016, pp. 10301033.

[5] J. Wolf and G. Ungerboeck, "Trellis Coding for Partial Response Channels" IEEE Transactions on Communications, vol. 34, no. 8, Aug 1986, pp. 765-773.

[6] H. C. Fereira and A. L. Hopenel, "Binary Rate Four Eighths, Runlength Constrained, Error Correcting Magnetic Recording Modulation Code" IEEE Transactions on Magnetics,vol. 22, no. 5, Sep 1986, pp. 1197-1199.

[7] P. Farkaš and H. Weinrichter, "Transcontrol Codes with RunLength Limitation" AEU Int. J. Electron. Commun., vol. 50, no. 6, 1996, pp. 353-356.

[8] M. Šechny and P. Farkaš, "Some New Runlength-limited Convolutional Codes" IEEE Trans. on Commun., vol. 47, no. 7, 1999, pp. 962-966.

[9] P. Farkaš, W. Pusch, M. Taferner and H. Weinrichter, "TurboCodes with Length Constraints" AEU Int. J. Electron. Commun., vol. 53, no. 3, 1999, pp. 161-166.

[10] P. Farkaš, "Turbocodes with RLL Properties" IEE Colloquium on Turbo Codes Digital Broadcasting Could it Double Capacity?, London, 22, November 1999, pp. 13/1-13/6.

[11] Y. Han and W. E. Ryan, "Concatenating a Structured LDPC Code and a Constrained Code to Preserve Soft-Decoding, Structure, and Burst Correction" IEEE Transactions on Magnetics, vol. 42, no. 10, Oct 2006, pp. 2558-2560.

[12] J. Lu and K. G. Boyer, "Novel RLL-ECC Concatenation Scheme for High-Density Magnetic Recording" IEEE Transactions on Magnetics, vol. 43 no. 6, June 2007, pp. 2271-2273.

[13] D. Hepper et al, "Increasing the Storage Density for a 4th-Generation Optical Disc" 2009 Digest of Technical Papers International Conference on Consumer Electronics, Las Vegas, NV, (2009), pp. 1-2.

[14] J. Kim, J. Lee and J. Lee, "Performance of Low-Density Parity Check Codes With Parity Encoded by $(1,7)$ Run-Length Limited Code for Perpendicular Magnetic Recording" IEEE Transactions on Magnetics, vol. 48, no. 11, Nov 2012, pp. 4610-4613.

[15] B. Vasic and K. Pedagani, "Run-Length-Limited Low-Density Parity Check Codes based on Deliberate Error Insertion" IEEE Trans. Magn., vol. 40. no. 3, May 2004, pp. 1738-1743.

[16] Li. Zongwang and B. V. K. V. Kumar, "Low-Density Parity-Check Codes with Run Length Limited (RLL) Constraints" IEEE Transactions on Magnetics, vol. 42, no. 2 Feb 2006, pp. 344-349.

[17] H. Y. Chen, M. C. Lin and Y. L. Ueng, "Low-Density Parity-Check Coded Recording Systems with Run-Length-Limited Constraints" IEEE Transactions on Magnetics, vol. 44, no. 9, Sep 2008, pp. 2235-2242.

[18] H. F. Chou, Y. L. Ueng, M. C. Lin and M. P. C. Fossorier, "An RLL-Constrained LDPC Coded Recording System Using Deliberate Flipping and Flipped Bit Detection" IEEE Transactions on Communications, vol. 60 no. 12 Dec 2012, pp. 3587-3596.
[19] P. Farkaš and F. Schindler, "Run Length Limited Error Control Codes Construction based on One Control Matrix Property" Journal of Electrical Engineering, vol. 68 no. 4, June 2017, pp. 322-324.

[20] R. G. Gallager, Low-Density Parity-Check Codes, MIT Press, Cambridge, 1963.

[21] A. J. Felstrom, and K. S. Zigangirov, "Time-Varying Periodic Convolutional Codes with Low-Density Parity-Check Matrix" IEEE Transactions on Information Theory, vol. 45, no. 6, Sep 1999, pp. 2181-2191.

Received 7 July 2017

Peter Farkaš is with Institute of Multimedia Information and Communication Technologies, Slovak University of Technology in Bratislava (STU) and also with Institute of Applied Informatics, Faculty of Informatics, Pan European University in Bratislava as a Professor. From 2002 till 2007 he was Visiting Professor at Kingston University, UK and senior researcher at SIEMENS PSE. In 2003 SIEMENS named him VIP for his innovations and patents. In 2004 he was awarded with the Werner von Siemens Excellence Award for research results on two-dimensional Complete Complementary Codes. From 2008 to 2009 he worked also as Consultant in the area of Software Defined Radio for SANDBRIDGE Tech. (USA). He was responsible leader of a team from STU in projects funded by the European Community under the 5FP and $6 \mathrm{FP}$ Information Society Technologies Programs: NEXWAY IST -2001-37944 (Network of Excellence in Wireless Applications and technology) and CRUISE (Creating Ubiquitous Intelligent Sensing Environments) FP6 IST-2005- 4- 027738, (2006-2007). His research interests include Coding, Communications Theory and sequences for CDMA. He published 1 book, 42 papers in reviewed scientific journals and 93 papers in international conferences. He is author or coauthor of 7 patents. He is and was serving in TPC of about 60 international conferences and presented 12 invited lectures. As an IEEE volunteer, Prof. Farkaš was serving in IEEE Czechoslovakia Section Executive Committee in different positions from 1992 to 2014 and from 2005 to 2006 he served as a chair of Conference Coordinator Subcommittee in IEEE Region 8. He organized IEEE R8 Conference EUROCON 2001 and was chairman of SympoTIC03, SympoTIC04, SympoTIC06 and co-organizer of Winter school on Coding and Information Theory 2005. From 2016 he serves as a vice-chair of Computer chapter in IEEE Czechoslovakia Section.

Frank Schindler Prof, Dr is a professor of Applied Informatics at the Faculty of Informatics of the Pan-European University in Bratislava. His education includes MSc (1978), Dr Nat Sci (1980) in Mathematics from Charles University, Prague, PhD (1983) in Mathematics from the University of Utah, Salt Lake City, Utah and Dr Habil (1994) in Informatics from Slovak Technical University, Bratislava. Besides that he has taught at the University of Maryland University College, University of Miami, University of Utah, Slovak Technical University and worked as software engineer for IBM Kingston, New York, Prime Computer Inc., Framingham, Massachusetts and Diagonal Systems AG, Zrich, Switzerland. Among his research interests are: computer graphics, geometrical modelling, object-oriented programming and computer security. 